This is an Accepted Manuscript of an article published by Taylor \& Francis in Medical Teacher on 11/03/17 available online:

http://www.tandfonline.com/doi/full/10.1080/0142159X.2017.1297531 


\title{
An exploration of teaching presence in online interprofessional education facilitation
}

\author{
Sherryn Evans, Catherine Ward, Scott Reeves
}

\begin{abstract}
Background: While the prevalence of online asynchronous interprofessional education (IPE) has increased in the last decade, little is known about the processes of facilitation in this environment. The teaching presence element of the Community of Inquiry Framework offers an approach to analyse the contributions of online facilitators, however to date it has only been used on a limited basis in health professions education literature.

Aim: Using an exploratory case study design, we explored the types of contributions made by IPE facilitators to asynchronous interprofessional team discussions by applying the notion of teaching presence.
\end{abstract}

Methods: Using a purposeful sampling approach, we analysed 14 facilitators' contributions to asynchronous team discussion boards in an online IPE course. We analysed data using directed content analysis based on the key indicators of teaching presence.

Results: The online IPE facilitators undertook the three critical pedagogical functions identified in teaching presence: facilitating discourse, direct instruction and instructional design and organisation. While our data fitted well with a number of key activities embedded in these three functions, further modification of the teaching presence concept was needed to describe our facilitators' teaching presence.

Conclusion: This study provides an initial insight into the key elements of online asynchronous IPE facilitation. Further research is required to continue to illuminate the complexity of online asynchronous IPE facilitation.

Keywords: interprofessional education; interprofessional learning; online; asynchronous; facilitation; teaching presence; community of inquiry framework; case study

\section{Introduction}

Over the past decade or so we have seen a rapid growth in the use of online learning methods in health professions education. Systematic reviews have found that online 
education is effective for improving attitudes, knowledge, skills and behaviours associated with professional competencies and practice (Cook et al. 2008; Jwayyed et al. 2011; Mącznik et al. 2015). This growth in online learning is also evident in the interprofessional education (IPE) arena, increasingly being used to overcome the scheduling and geographical challenges associated with the delivery of traditional face to face IPE (e.g. Casimiro et al. 2009; Evans et al. 2013). While a range of online synchronous and asynchronous interprofessional activities have been reported, online asynchronous team discussion boards have been most commonly used (Curran et al. 2015). A growing body of literature indicates that the use of such online asynchronous learning activities can result in positive outcomes for IPE participants (e.g. Evans et al. 2013; Solomon et al. 2010; Curran et al. 2015).

However, less is known about the contribution of facilitators in online asynchronous IPE. In the asynchronous online discussion environment the facilitation process is complex with facilitators needing to ensure participants actively engage in the dialogue, discussion and debate that is essential for interprofessional learning (Reeves et al. 2016), all without the important non-verbal and paralinguistic cues available with traditional forms of facilitation. While a small number of researchers have started to explore aspects of online asynchronous IPE facilitation (e.g. Solomon \& King 2010; Evans et al. 2014; Evans et al. 2016), relatively little attention has been given to the actual contributions facilitators make in an asynchronous online IPE environment.

Outside of the health arena, the contributions of the facilitator in online asynchronous learning has been explored in some more detail. An early example, the Community of Inquiry (Col) framework, introduced by Garrison et al. (2000) is a model that consists of three interdependent elements (cognitive presence, social presence and 
teaching presence) that contribute to successful online asynchronous teaching/learning. Anderson and colleagues (2001) defined teaching presence as "the design, facilitation, and direction of cognitive and social processes for the purpose of realizing personally meaningful and educationally worthwhile learning outcomes" (p 5). Teaching presence, summarised in Table 1, consists of three categories (representing critical functions) of the online teacher: instructional design and organization, facilitating discourse and direct instruction (Anderson et al. 2001). Each of these categories include a number of indicators that help define their function. Collectively, they can be used as a template to analyse online asynchronous dialogue, along with providing guidance on the optimal use of online discussions (Garrison et al. 2000).

\section{Insert table 1 here}

The notion of teaching presence has been examined in a number of studies across a range of different contexts (e.g. Arbaugh \& Hwang 2006; Shea et al. 2006). Its application in the health professions education literature however is much more limited, with two papers that have used this concept (Kamin et al. 2006; Waterston 2011), only one of which, Waterston (2011), employing the concept in an online IPE context. This study found that student teams with positive perceptions of their online IPE experiences had facilitators that more frequently encouraged participation (an indicator of facilitating discourse) and presented content and asked questions (an indicator of direct instruction). Given the lack of literature in this area, further investigation is needed. The current paper reports the findings from a study that explored the contributions made by IPE facilitators to asynchronous interprofessional team discussions related to teaching presence.

\section{Background}


The Faculty of Health at [removed for blind review], Australia, runs an online IPE unit (comprising 110 hours of study over a 11-week period) utilising asynchronous and synchronous communication technology for pre-licensure students from seven different health professions (clinical exercise physiology, nursing, occupational therapy, social work, medicine, dietetics and psychology) (see [removed for blind review]) for a detailed overview of the unit). One component of this unit involves students being allocated to interprofessional teams of eight students. Each team is guided by a facilitator: a qualified health professional who has worked in collaborative practice environments. Each week the facilitators guide these teams in online asynchronous discussions that focus on issues related to collaborative practice along with the development of interprofessional team care plans. At the start of each week, facilitators upload a new topic for discussion on to their team discussion board. This initial message is pre-scripted by the teaching team, thereby setting the curriculum for that week. After this initial message, the facilitators are encouraged to contribute to the discussion approximately every second day to ensure active involvement in the discussion, however the content of these contributions is not prescribed. Facilitators volunteer to lead either one, two or three student teams for the duration of the course. Prior to commencement of the unit, facilitators undertake online training that addresses the content of the unit, the use of the various programs, and their role as a facilitator of learning.

\section{Methods}

\section{Design}

This study employed an exploratory case study design (Yin 2002) and gathered data on facilitators' contributions to an asynchronous online interprofessional course based at 
[removed for blind review] University. The study aimed to explore the types of contributions made by IPE facilitators to asynchronous interprofessional team discussions by using Anderson and colleagues' (2001) notion of teaching presence.

\section{Data collection}

Following ethical approval from the University's Ethics Research Committee, all facilitators from the online IPE unit $(n=28)$ were invited by email to provide their consent to have their online teaching contributions from the previous trimester of study downloaded and analysed. Following the facilitators return email providing their consent, a research assistant downloaded the facilitators' contributions from the online discussion boards. Facilitator information was de-identified during this process to protect participant anonymity. All references to students' names were also removed during the process.

All 28 facilitators involved in the unit responded to the email. The facilitator cohort consisted of seven physiotherapists, six occupational therapists, six social workers, four nurses, three dietitians, one speech pathologist and one psychologist. Three facilitators were male and 25 were female. Seventeen of the facilitators led one student, nine led two student teams, whereas two facilitators led three teams. The participants had between one and six years' experience in online IPE facilitation, and none had facilitated IPE in a face-toface context. All were sessional university teaching staff employed specifically for the facilitation role. All but one were also practicing as a health professional.

A purposeful sample of the facilitators who led one team of students was identified from the data set for full analysis. For consistency of facilitation experience, it was decided to focus the sample on those facilitators who led one student team during the course. These 14 facilitators included three physiotherapists, three occupational therapists, three social 
workers, three dietitians, one speech pathologist and one psychologist; one was male and 13 were females. For four of these facilitators it was their first year of online IPE facilitation, while the others all had between two and six years' experience.

\section{Analysis}

Transcripts of the 14 facilitators' online contributions were analysed (approximately 500 pages of data) using a directed content analysis linked to teaching presence (see Table 1). The remaining 14 facilitator's transcripts, those not sampled for full analysis, were subsequently used to help verify the emergent analysis.

All three researchers initially coded one of the transcripts to generate a consensus about analysis. Once agreed, two researchers independently coded the transcripts, meeting regularly to clarify and ensure consistency of the analysis, and discuss any emergent themes or ambiguity.

Four indicators emerged during the analysis which would not 'fit' into the existing framework. As a result, we created three new indicators in the direct instruction category (presenting personal reflections, reminding students about learning tasks and feedback on assessment tasks); and one new indicator in the facilitating discourse category (informal/social elements). Each of these new indicators were added into the framework see Table 2.

\section{Results}

This section presents results in four sections. First we offer an insight into the extent of the facilitators' online contributions. We then go on to present our findings in relation to 
the three categories of teaching presence: facilitating discourse, direct instruction and instructional design and organisation.

\section{Extent of facilitators' contributions}

Our analysis revealed that the 14 facilitators posted, on average, 64 messages on their team discussions, with a minimum of 43 being posted by one facilitator and a maximum of 90 by another. In total, the 14 facilitators posted 899 messages during the course.

Within this total number of messages, we identified 2118 individual facilitator contributions which we coded into the different indicators of teaching presence. Table 2 summarises frequencies for these indicators.

As presented in Table 2, half of facilitators' contributions were coded into the 'facilitating discourse' category. Approximately a third were coded in to 'direct instruction' category, with the remainder in the 'instructional design and organisation' category. Below we present findings, with representative quotes from the facilitators, from the more frequently-used indicators as well as the four newly created indicators.

\section{Insert table 2 here}

\section{Facilitating discourse}

As Table 2 shows, the most commonly used indicator was 'encouraging, acknowledging or reinforcing student contributions'. Many facilitators began their messages with this approach, often simply acknowledging student posts related to interprofessional collaboration. As the following data extract illustrates: 
"Thanks for joining the conversation $X$ and $X$ [students] - you both raise some really important characteristics of collaborative teams. Thanks for sharing your experiences." (Facilitator 8)

In addition to acknowledging the students' responses, the facilitators often also encouraged the students on the applicability of their contributions to interprofessional learning:

"Thanks for sharing your thoughts and reflections on professional overlap. I really appreciated your reflection on a situation that you would do differently next time round and what you learnt from the experience. It was a really good example of the potential overlap issues." (Facilitator 3)

In acknowledging the students' contributions, facilitators often reinforced the significance of the students' contribution to key factors of collaborative practice, such as communication, conflict resolution and patient-centred care:

"The last comment made by $X$ [student] is very significant. So much informal case discussion takes place when health professionals are in close proximity - quick corridor chats so to speak (although in an area that is private!) and these discussions usually help to address issues early and keep things moving along." (Facilitator 15)

The second most frequently used indicator was 'drawing in participants, prompting discussion" (Table 2). Statements such as "looking forward to your thoughts" (facilitator 10) and "interested to continue to hear your perspectives on this one" (facilitator 17) were regularly used by the facilitators to prompt the students to continue to participate in the discussion on the topic. In addition, facilitators regularly targeted specific student team members in their messages to draw them into the interprofessional conversation if they had not contributed at all or minimally:

"There are a few team members we would benefit from hearing from in this thread to ensure their contributions are represented, OT and nursing in particular, so if you have some ideas, please post them asap." (Facilitator 9) 
Another frequently used indicator was 'setting climate for learning'. Facilitators often conveyed that all students input was valuable and that it was a "safe" interprofessional environment for them to learn in, as indicated in this data extract:

"On that note team - don't be shy team with your contributions to the second discussion thread-you all have lots to offer and we can all learn from each other. I would love to hear your perspective." (Facilitator 9)

One of the new indicators related to facilitating discourse, 'informal/social elements', capturing facilitators' awareness of students social lives. At times, facilitators included contributions such as "good luck in your netball final" (facilitator 12) and "I am wondering if you all had a great Easter long weekend" (facilitator 18).

\section{Direct Instruction}

One of the new indicators in the direct instruction category, 'presenting personal experiences/reflections', was commonly used to highlight that facilitators contributed their personal reflections on interprofessional collaboration in healthcare to the discussions. These personal insights were used by facilitators to spark the students' engagement in the discussions by role modelling appropriate reflective contributions, or to be an active participant in an already flourishing teams' conversation:

"So many times I have sat with patients [as a dietitian] and shown them a picture of the GI tract and explained the role of different parts of the bowel in nutrient absorption, and how this will now be impacted by their surgery. I never minded that the surgeons did not do it - they knew I did, and that I had time to sit and do it properly. I can't operate, or prescribe, or medically / surgically manage patients, so I guess I did my bit and they did their bit? It worked well, because we had mutual respect and understanding for each other's role." (Facilitator 18)

Data coded into the indicator, 'summarise the discussion' represented facilitators drawing together points raised by a number of students in preceding discussions. All 
facilitators did this in their last post for each topic. Some facilitators, however, also summarised mid-topic to highlight the depth of conversation occurring along with commonalities in their contributions:

"Thank you [student names] for building on those previous discussions. Although all health care related, we are seeing diverse examples from placements to previous professional roles encompassing community health, rehab and acute care. Commonalities in your discussions included that for effective collaboration, common/shared goals, mutual respect and good communication are important." (Facilitator 14)

Another indicator, 'present content/question', revealed that facilitators often presented content in their contributions, usually to stimulate further discussion on specific points. This content was usually presented as some brief content on a topic, followed by a question for the students to consider and respond to:

"Communication is so important here, not just from the leader but from each of the team members. Even from the perspective of a quieter team member, yes your colleagues can modify their approach to ensure your inclusion, but what is your responsibility to the team, how will you contribute? Sometimes when we are new to a practice environment we can feel reserved, quiet, unsure and lack to the confidence to speak up. What strategies can you adopt to modify your own presentation?." (Facilitator 12)

Another of the newly emerged indicators, 'reminding students about learning activities' related to the facilitators tendency to frequently remind students about key aspects of the learning activities of their online interprofessional course. These reminders were often about tasks the students needed to be actively involved in, with a particular emphasis on the importance of this participation given some of their assessment was dependent upon it:

"I just want to remind you all to add your bits to the [interprofessional] care plan and save back onto this thread so it is not all left to $X$. It is everybody's responsibility to 
support this care plan development. Please remember contributing to threads and care planning is a big part of your mark." (Facilitator 7)

The third newly emerged indicator, 'feedback on assessment tasks' related to a tendency for facilitators to provide both formative and summative feedback on assessment tasks students were developing in the discussion threads. Formative feedback was most commonly provided to the teams in a specific task in which they developed interprofessional care plans on the discussion boards:

"I understand that this is still a work in progress, but just a reminder to separate goals from actions and to keep those goals patient centred. Also, consider if the goal is measurable (not always truly possible in health care, but do it where possible)." (Facilitator 27)

In contrast, summative feedback was provided on the discussion boards based on the finalised care plan that was submitted by the student teams for assessment purposes:

"Congratulations on your first care plan. This was a great effort and your thorough plan reflects a team that is working well together. Please find your mark and feedback attached." (Facilitator 9)

\section{Direct instructional design and organisation}

While this was the least frequently used category of teaching presence in this study

(Table 2), two indicators were still regularly used: 'establishing time parameters' and 'utilising medium effectively'.

In the early weeks of their role, facilitators particularly concentrated on 'establishing time parameters' related to negotiating when a specific synchronous learning activity would occur and reminding them about these times. As outlined in the following data extract:

"It seems like we are so far all free on Mon or Thurs, so let's wait for the last 2 team members to jump on board and see what they say." (Facilitator 18) 
Over time, this shifted to the facilitators focusing more on when discussion topics would be closed to the students and when assessment tasks were due.

Facilitators often used the indicator 'utilising medium effectively' when referring to appropriate use of the online asynchronous discussion medium to ensure detailed interactive dialogue would occur among the students. Facilitators would encourage the students to build on the content of each other's posts and to ensure that the thread become a "conversation" rather than a collection of individual ideas:

"Please remember that if you have posted once please feel free (and be encouraged!) to do so again to elaborate more on what you and others have said so as to continue in the conversation". (Facilitator 5)

At other times, this was reinforcement for the students doing this well:

"As an aside, your discussion showcased a good example of building on and reflecting on other's postings, which is an important part of this unit- so thank you".

(Facilitator 14)

\section{Discussion}

This paper presented the findings from a study that aimed to explore the types of contributions made by IPE facilitators to asynchronous interprofessional team discussions using Anderson and colleagues' (2001) notion of teaching presence. We found that the teaching presence provided a helpful conceptual lens to understand how teaching presence for IPE facilitators related to their contributions while guiding online asynchronous team discussions.

Specifically, the study found that facilitators used indicators included in each of the three categories of teaching presence presented by Anderson and colleagues (2001); facilitating discourse, direct instruction and instructional design and organisation. This suggests that these online IPE facilitators were undertaking, at least in part, the three critical functions of an online facilitator. However, from the 18 indicators included in the three categories of teaching presence outlined by Anderson and colleagues (2001), only the 
following seven were frequently used by the facilitators in the current study: 'encouraging, acknowledging, or reinforcing student contributions', 'drawing in participants, prompting discussion', 'setting climate for learning', 'summarise the discussion', 'present content' from the direct instruction category, 'establishing time parameters' and 'utilising medium effectively' (Table 2).

The remaining 11 indicators from the teaching presence model were not frequently used by facilitators in the current study. In some cases, this may be an indication of a facilitative approach within this IPE context. For example, the facilitators were trained to not use a traditional didactic teaching style but rather use a "facilitation" style, aiming to guide and assist students in their own learning. Based on this premise, direct instruction such as "diagnosing misconceptions" and "injecting knowledge from diverse sources" for example, may have seemed too directive for these facilitators. The minimal use of other indicators, such as "establishing netiquette" for example, may reflect a degree of "ageing" of the teaching presence notion. Anderson and colleagues' (2001) indicators were developed over 15 years ago, well before the significant growth in the digital age. As a result, current learners have grown up with the use of online technologies and are likely to be very familiar with netiquette, therefore not requiring our facilitators to establish boundaries around this. Alternatively, the minimal use of some indicators may simply be a reflection of the specific asynchronous learning discussion tasks the students were involved in in our course, or a coding bias of the researchers.

While our data did not fit into a number of the original indicators, we did identify three new indicators that our facilitators regularly used (a fourth new one, informal/social elements, was identified but not regularly used). These new indicators reflected unique aspects of our facilitators' contributions that were not captured by Anderson and colleagues 
(2001). The first and most commonly used of our new indicators was 'presenting personal reflections'. The original framework included indicators of 'present content/questions' and 'inject knowledge from diverse sources' but neither of these indicators represented our data linked to the unique personal experiences and reflections our facilitators were providing. The IPE facilitators in this study used these reflections to trigger the students to engage in the discussions by role modelling contributions, or to simply be an active participant in the teams' discussion. With IPE being deeply grounded in adult learning theory, reflective learning is a well-recognised key component of IPE curriculum (Freeth et al. 2005). Given the importance of reflection in IPE, it therefore accords well that a reflective approach is modelled by IPE facilitators.

Our analysis also resulted in adding the indicator 'feedback on assessment tasks' to reflect the formative and summative contributions facilitators were providing related to assessment tasks students were developing and submitting on their discussion threads. The indicator 'reminding students about learning activities' was also added from our analysis, which often included facilitators reminding students that participating in these activities was important for assessment purposes. This analysis supports recent work which has used the teaching presence element of the COI framework and found that "assessment" might in fact be an additional fourth category of teaching presence (Shea et al. 2010). Like our work, these suggested that this may relate to both formative and summative feedback on assessment tasks.

Combined, these three frequently used new indicators, along with the seven frequently used indicators from Anderson and colleagues (2001) original model, help provide an initial insight into the key elements of online asynchronous IPE facilitation. For interprofessional learning to occur, participants need to actively engage in dialogue, 
discussion and debate (Howkins \& Bray 2008). The role of the IPE facilitator therefore involves encouraging participant interaction and group cohesion, and leading the group process to meet diverse learning needs (Freeth et al. 2005; Derbyshire et al. 2015) . This study is a step towards further defining the role of the IPE facilitator more specifically in the online context.

In relation to study limitations, the small sample of facilitators involved in this study combined with the fact that the study was undertaken at a single institution will restrict the transferability of our findings to other contexts. Our future work will attempt to include online asynchronous IPE discussions from other institutions to assess the transferability of these findings. Further, as the data gathered focused only on the facilitators' contributions, our study does not provide an insight into student reaction. Exploring the relationship between facilitator contributions and student contributions and their perceptions of the IPE experience will further build on the insights from this study - this will be the focus of a future paper.

Despite these limitations, this study provided a rare empirical insight into the nature of online asynchronous IPE facilitation. Using the concept of teaching presence, it found that three pedagogical functions of facilitating discourse, direct instruction and instructional design/organisation were key to this form of IPE facilitation. While a number of the activities associated with these functions fitted our data well, some modification was needed for an IPE online context. However, additional research is needed to further understand the complex nature of online asynchronous IPE facilitation.

\section{Practice Points}


- The facilitation of online asynchronous IPE is complex, yet little research has been undertaken into the complexities of this role.

- The teaching presence element of the Community of Inquiry Framework can be employed to provide a useful lens to explore key elements of online asynchronous IPE facilitation.

- Key teaching presence functions related to online asynchronous IPE facilitation focused on facilitating discourse, direct instruction and instructional design and organisation.

- Future research is needed to further understand the complexity of online asynchronous IPE facilitation.

\section{Acknowledgement}

The study was approved by Deakin University Human Research Ethics Committee (HEAG-H 129-2014).

\section{Declaration of Interest}

The authors report no conflict of interest.

\section{References}

Anderson T, Rourke L, Garrison R, Archer W. 2001. Assessing teaching presence in a computer conferencing context. JALN, 5, 1-17.

Arbaugh JB, Hwang A. 2006. Does "teaching presence' exist in online MBA courses? The Internet and Higher Education, 9, 9-21.

Casimiro L, Macdonald, CJ, Thompson TL, Stodel EJ. 2009. Grounding theories of W(e)Learn: a framework for online interprofessional education. Journal of Interprofessional Care, 23, 390-400.

Cook D, Levinson A, Garside S, Dupras D, Erwin P, Montori V. 2008. Internet-based learning in the health professions: a meta-analysis. JAMA, 300, 1181-1196.

Curran V, Deacon D, Fleet L. 2005. Academic administrators' attitudes towards interprofessional education in Canadian schools of health professional education. Journal of Interprofessional Care, 19, 76-86. 
Derbyshire JA, Machin Al, Crozier S. 2015. Facilitating classroom based interprofessional learning: a grounded theory study of university educators' perceptions of their role adequacy as facilitators. Nurse Education Today, 35, 50-56.

Evans S, Knight T, Sønderlund A, Tooley G. 2014. Facilitators' experience of delivering asynchronous and synchronous online interprofessional education. Medical Teacher, $36,1051-1056$.

Evans S, Shaw N, Ward C, Hayley A. 2016. Refreshed...reinforced...reflective: A qualitative exploration of interprofessional education facilitators' own interprofessional learning and collaboratie practice. Journal of Interprofessional Care.

Evans S, Sonderland A, Tooley G. 2013. Effectiveness of online interprofessional education in improving students' attitudes and knowledge associated with interprofessional practice. Focus on Health Professional Education, 14, 12-20.

Freeth D, Hammick M, Reeves S, Koppel I, Barr H. 2005. Effective interprofessional education: development, delivery and evaluation, Oxford, UK ; Malden, MA, Blackwell Pub.

Garrison DR, Anderson T, Archer W. 2000. Critical inquiry in a text-based envrionement: Computer conferencing in higher education. The Internet and Higher Education, 2, 87-105.

Howkins E, Bray J. 2008. Preparing for Interprofessional Teaching: Theory and Practice. Oxford, Radcliffe Publishing.

Jwayyed S, Siffler KA, Wilber ST, Southern A, Weigand J, Bare R, Gerson LW. 2011. Technology-assisted education in graduate medical education: a review of the literature. International Journal of Emergency Medicine, 4, 51.

Kamin CS, O'sullivan P, Deterdin RR, Younger M, Wade T. 2006. A case study of teaching presence in virtual problem-based learning groups. Medical Teacher, 28, 425-8.

Mącznik AK, Ribeiro DC, Baxter GD. 2015. Online technology use in physiotherapy teaching and learning: a systematic review of effectiveness and users' perceptions. BMC Medical Education, 15, 160-160.

Shea P, Chun Sau L, Pickett A. 2006. A study of teaching presence and student sense of learning community in fully online and web-enhance college courses. Internet and Higher Education, 9, 175-190.

Shea P, Vickers J, Hayes S. 2010. Onlineinstructional effort measured through thelens of teaching presence in the community of inquiry framework: A re-examination of measures and approach. . The International Review of Research in Open and Distance Learning, 11. 
Solomon P, Baptiste S, Hall P, Luke R, Orchard C, Rukholm E, Carter L, King S, Damiani-Taraba G. 2010. Students' percpetions of interprofessional learning through facilitated online learning modules. Medical Teacher, 32, e391-e398.

Solomon P, King S. 2010. Online interprofessional education: perceptions of faculty facilitators. Journal of Physical Therapy Education, 24, 51-53.

Waterston R. 2011. Interaction in online interprofessional education case discussions. Journal of Interprofessional Care, 25, 272-279.

Yin RK. 2002. Case study research: Design and methods. Thousand Oaks, CA, Sage Publications. 
Table 1: Teaching presence categories and indicators (Anderson et al. 2001)

\begin{tabular}{|l|l|}
\hline Categories & Indicators \\
\hline Instructional Design & Setting curriculum \\
\cline { 2 - 2 } & Designing methods \\
\cline { 2 - 2 } & Establishing time parameters \\
\cline { 2 - 2 } & Utilizing medium effectively \\
\cline { 2 - 3 } & Establishing netiquette \\
\hline Facilitating discourse & Identifying areas of agreement/disagreement \\
\cline { 2 - 3 } & Seeking to reach consensus/understanding \\
\cline { 2 - 3 } & Encouraging, acknowledging, or reinforcing student contributions \\
\cline { 2 - 3 } & Setting climate for learning \\
\cline { 2 - 3 } & Drawing in participants, prompting discussion \\
\cline { 2 - 3 } & Assess the efficacy of the process \\
\hline Direct Instruction & Present content/questions \\
\cline { 2 - 3 } & Focus the discussion on specific issues \\
\cline { 2 - 3 } & Summarize the discussion \\
\cline { 2 - 3 } & Confirm understanding through assessment and explanatory feedback. \\
\cline { 2 - 3 } & Diagnose misconceptions \\
\cline { 2 - 3 } & $\begin{array}{l}\text { Inject knowledge from diverse sources, e.g., textbook, articles, internet, } \\
\text { personal experiences (includes pointers to resources) }\end{array}$ \\
\cline { 2 - 3 } & Responding to technical concerns \\
\hline &
\end{tabular}


Table 2: Frequency of teaching presence indicators for sampled facilitators

\begin{tabular}{|c|c|c|c|}
\hline Category & Indicators & $\begin{array}{l}\text { Facilitator } \\
\text { contribution }\end{array}$ & $\begin{array}{l}\text { Percentage of } \\
\text { total } \\
\text { facilitator } \\
\text { contributions }\end{array}$ \\
\hline \multirow[t]{8}{*}{$\begin{array}{l}\text { Facilitating } \\
\text { discourse }\end{array}$} & $\begin{array}{l}\text { Encouraging, acknowledging, or reinforcing } \\
\text { student contributions }\end{array}$ & 691 & 32.6 \\
\hline & Drawing in participants, prompting discussion & 283 & 13.8 \\
\hline & Setting climate for learning & 60 & 2.8 \\
\hline & Informal/social elements* & 12 & 0.6 \\
\hline & Assessing the efficacy of the process & 5 & 0.2 \\
\hline & Identifying areas of agreement/disagreement & 3 & 0.1 \\
\hline & Seeking to reach consensus/understanding & 3 & 0.1 \\
\hline & Subtotal & 1057 & 50.2 \\
\hline \multirow[t]{11}{*}{$\begin{array}{l}\text { Direct } \\
\text { Instruction }\end{array}$} & Presenting personal reflections* & 163 & 7.7 \\
\hline & Summarize the discussion & 162 & 7.6 \\
\hline & Present content/questions & 104 & 4.9 \\
\hline & Reminding students about learning activities* & 101 & 4.8 \\
\hline & Feedback on assessment tasks* & 101 & 4.8 \\
\hline & Focus the discussion on specific issues & 21 & 1.0 \\
\hline & Responding to technical concerns & 16 & 0.8 \\
\hline & $\begin{array}{l}\text { Confirm understanding through assessment } \\
\text { and explanatory feedback }\end{array}$ & 11 & 0.5 \\
\hline & $\begin{array}{l}\text { Inject knowledge from diverse sources, e.g., } \\
\text { textbook, articles, internet, (includes pointers } \\
\text { to resources) }\end{array}$ & 8 & 0.4 \\
\hline & Diagnose misconceptions & 7 & 0.3 \\
\hline & Subtotal & 694 & 32.8 \\
\hline
\end{tabular}




\begin{tabular}{|l|l|r|r|}
\hline $\begin{array}{l}\text { Instructional } \\
\text { Design and } \\
\text { Organisation }\end{array}$ & Establishing time parameters & 271 & 12.8 \\
\cline { 2 - 4 } & Utilizing medium effectively & 50 & 2.3 \\
\cline { 2 - 4 } & Setting curriculum (including assessment) & 24 & 1.1 \\
\cline { 2 - 4 } & Designing (learning) methods & 16 & 0.8 \\
\cline { 2 - 4 } & Establishing netiquette & 6 & 0.2 \\
\cline { 2 - 4 } & Subtotal & $\mathbf{3 6 7}$ & $\mathbf{1 7 . 2}$ \\
\cline { 2 - 4 } & Totals & $\mathbf{2 1 1 8}$ & $\mathbf{1 0 0}$ \\
\hline
\end{tabular}

Key: * denotes a new indicator added which emerged from the analysis 